\title{
Reliable Guaranteed Cost Control of Networked Control System with Var- iable Sampling Period
}

\author{
Nan $\mathrm{Xie}^{*}$ and Bin Xia
}

School of Computer Science and Technology, Shandong University of Technology, Zibo255049, China

\begin{abstract}
This paper discusses the reliable guaranteed cost control problem of networked control systems with timevarying sampling period and actuator failures for a given quadratic cost function. For linear time-invariant controlled plant, we assume that the time delay is constant and shorter than the sampling period, the sampling is time-varying within a certain known bound, the system is transformed into a time-varying discrete time system, and where the time-varying sampling period parts are considered as norm bounded uncertainties of the structure parameter. Based on the linear matrix inequality (LMI) method, we have derived a sufficient condition for the existence of a reliable guaranteed cost controller. Furthermore, we have also designed a convex optimization problem with LMI for optimal reliable guaranteed cost controller which can decrease the upper bound of the closed-loop system cost. A numerical example is given to illustrate the proposed method.
\end{abstract}

Keywords: guaranteed cost control, LMI, Networked Control System (NCS), reliable, variable sampling period.

\section{INTRODUCTION}

Recently, owing to the expansion of physical setups and functionality, networked control systems (NCSs) have been introduced into the design of control systems. NCSs are distributed control systems in which sensors, actuators, and controllers are interconnected through communication networks. The use of NCSs have rapidly increased in the industrial sector because of its low cost, flexibility, and a smaller amount of wiring. Therefore, researchers are paying greater attention to the study of NCSs $[1,2]$. As we know that the sampling data and controller signals are communicated through a network, the delays and data dropouts in the NCSs are almost inevitable. The network-induced delays may be constant, time-varying, or even random for the NCSs with different scheduling protocols. In the recent past, several researchers have investigated the stability analysis and stabilization controller design for NCSs when the effects of network-induced delay and/or data dropout are taken into account [3-6].

Several papers have been published by leading researchers who have studied the problem of varying sampling period of networked control systems [7, 8]. In case of the NCSs, the system performance is better when the sampling period is shorter. However, there is a possibility of network congestion if the sampling period is short. Wang and Yang [8] use the novel active-varying sampling period method to eliminate the possibility of packet disordering, which significantly streamlines the design and analysis of NCSs, and ensures the appropriate use of bandwidth when the network is idle. The conditions of robust stability of the NCSs with stochastic disturbance are given in [9], and Gao et al. [10] transforms the stochastic sampling system into a continuous time-delay system, and study the robust $H_{\infty}$ control problem. [11] designs the nonstatic observer and state controller with no considerations of the disturbance and noise, and discusses the application condition of the separation principle. Liu et al. [12] use the dynamic interval principle to design the corresponding controller, but the proposed algorithm is complicated and needs great amount of computation. M. Izak et al. have studied the stability and control related problems with uncertain and time-varying sampling period and time-delay [13]. In the discretized system description, uncertain and time-varying sampling period and delays are converted into polytopic and additive norm-bounded uncertainties. Y. Xu et al. have modeled the random sampling measurement process caused by the time-varying channel load as a Markov chain [14], which is an event-driven transmitter mainly depending on the measurement sampling period introduced for transmitting the control signal.

The optimal control for nonlinear and/or time-delay systems is one of the most active subjects in control theory. Besides the simplest cases, the optimal state feedback control problems do not exist analytical solutions for quadratic cost functional of nonlinear and/or time-delay systems. G.-Y. Tang has proposed the successive approximation approach (SAA) designing optimal controllers for kind of nonlinear systems with a quadratic performance index $[15,16]$. By applying the SAA, the nonlinear optimal control system is converted into a series of nonhomogeneous linear two-point boundary value (TPBV) problems. The optimal control law consists of an accurate linear feedback term and a nonlinear compensation term which is the limit of an adjoint vector sequence. 
The problem of designing robust controllers for systems with parameter uncertainties has attracted significant attention in recent control system literature [17-20]. It has also been suggested to design a stable control system which also guarantees a satisfactory level of performance. One approach to this problem is the so-called guaranteed cost control approach first introduced by Chang and Peng [21]. This method has the benefit of providing the upper bound on a defined performance index and therefore the degradation in the system performance incurred by the uncertainties are confirmed to be smaller than this bound. Some important results have been proposed for the continuous-time case [22, 23] and for the discrete-time case [24] based on the above mentioned studies.

Actuators play an important role in relaying the controller output to the plant. Actuator failures may be encountered sometimes. Furthermore, how to preserve the closed-loop system performance in the case of actuator failures will be tougher and more meaningful. Efforts have been put in to solve the problem related to reliable guaranteed cost controller design which have produced some good results for the continuous-time case [25] and the discrete-time case [26]. However there are very few results reported in the available literature on the reliable guaranteed cost controller design of nonlinear discrete-time systems with time-varying state delay and actuator failure.

There are a number of patents considering the problem of networked control systems in the last a few years. Patent US $8,611,220$, titled "Network system, controller, and network control method" [27], provides when an appliance, which has a packet discard function, discards a packet, transmits packet discard information as information related to the discarded packet to a controller. A controller performs a switch control process in response to the packet discard information. In the control process, the controller selects a target switch from the network, creates entry setting information for instructing the target switch to create an entry such that a packet belonging to the same flow as the discarded packet is discarded, and transmits the created entry setting information to the target switch. The target switch sets an entry in its own flow table in response to the entry setting information transmitted from the controller. In Patent US $8,422,359$, titled "Communication network control system and control method" [28]. A case of failure of fabric management mechanism has been reported nodes resumed data $\mathrm{I} / \mathrm{O}$ communications without deteriorating performance and without changing the data $\mathrm{I} / \mathrm{O}$ communication path between the nodes by switching control to other of the fabric management mechanisms. The fabric management mechanisms share management information with each other. When a failure occurs in either of the fabric management mechanisms, an E_Node that belongs to the domain, in which a failure has occurred, logs into a normal fabric management mechanism via a newly created management-use communication path. The normal fabric management mechanism allocates an N_Port_ID on the basis of a virtual FC domain number that has been allocated to a switch. In Patent US $8,218,547$, titled "Networked control system using logical addresses" [29], networked control systems and methods include a plurality of devices connected to a network are included. At least one device has a logical address that in- cludes a device type and a device location in an environment of the device in a user readable and understandable format, such as English or other languages. The system further includes a processor connectable to the network and configured to execute control logic using the logical addresses. A memory is provided for storing a mapping from the logical address to a physical network address of the device. In Patent US $8,335,156$, titled "Network connection control system" [30], a network connection control system is provided with: a relay device that relays communication in a communication network; and a communication terminal that performs communication via the relay device. The relay device has a network information transmission section, which is information about the communication network. The communication terminal has: a lower communication section that performs communication via the relay device; a network information receiving section that receives the network information via the lower communication section; a higher communication section that performs interhigher-section communication via the lower communication section, with another higher communication section in another communication terminal; a judgment result acquisition section that acquires a judgment result based on the received network information, about whether or not to permit the inter-higher-section communication; and a communication control section that inhibits the inter-higher-section communication until a judgment result that the inter-higher-section communication is to be permitted is acquired. In Patent US $8,477,601$, titled "Network management station, network control system, and network management method" [31], the present invention enables dynamic route switching according to link quality, while maintaining the quality of a plurality of routes. According to quality information received from a second network control entity, if the control unit of a network management station has determined that the quality of communication over a particular link has degraded, the control unit assigns a link whose current throughput satisfies a bandwidth required for a service defined in the service contract and whose response time satisfies minimum response time defined in the service contract to each service in descending order of priority. In Patent US 8,396,060, titled "Network control system" [32], a network control system for packet-transmitting a manipulated variable (MV), calculated from a deviation between a variable from a sensor measuring a physical quantity of a controlled object plant and a set value at a known sampling period, to an actuator provided on the plant side via a network, and causing the actuator to provide the MV to the plant. The system includes: an MV complementing unit that provides a complementary value of the MV to the actuator when an error occurs in a packet transmission; an MV buffering unit that answers back trend data of the MV provided to the plant from the actuator or the complementary value being complemented by the MV compensating unit for the actuator, to the controller via the network; and an MV compensating unit that corrects a calculation of the MV of the controller based on the trend data or the complementary value being answered. In Patent US 8,447,334, titled "Network control device and network control method" [33], as a network control device which controls a call connection between a calling party terminal and a called party terminal, a network control device determines whether the own network control device is in a 
congestion state when a handover request is acquired from another network control device which is in the congestion state, and stops transmitting a busy tone to a mobile terminal which is the calling party terminal and transmits a ring back tone to the mobile terminal when it is determined that the own network control device is not in the congestion state.

In section 2 of this paper, we assume that for linear timeinvariant controlled plant the time delay is constant and shorter than the sampling period, the sampling is timevarying during a definite known bound, the system is transformed into a time-varying discrete time system in which the time-varying sampling periods are treated as norm bounded uncertainties of the structure parameter. In section 3, based on the linear matrix inequality (LMI) method, a suitable condition for the presence of reliable guaranteed cost controllers is derived. In addition, we formulate a convex optimization problem with LMI constraints for designing optimal reliable guaranteed cost controller which reduces the upper bound of the closed-loop system cost. We have proposed the reduction algorithms for decreasing the required amount of LMIs needed for stability analysis. In section 4, the control design and the reduction algorithm are illustrated by an example.

\section{PROBLEM STATEMENT}

Consider the following networked control system

$\left\{\begin{array}{l}\dot{x}(t)=\mathrm{A}_{c} x(t)+\mathrm{B}_{c} u(t) \\ y(t)=\mathrm{C}_{c} x(t)\end{array}\right.$

where $\quad x(t) \in R^{n} \quad$ is the state vector, $u(t)=\left[\begin{array}{llll}u_{1}(t) & u_{2}(t) & \cdots & u_{m}(t)\end{array}\right]^{\mathrm{T}} \in R^{m}$ is the control vector, and $y(t)$ is the output vector.

Define $\tau_{S C}^{k}$ and $\tau_{C A}^{k}$ as the sensor-to-controller and controller-to-actuator network transmission time delays, and $\tau_{C}^{k}$ is the computation time delay of actuator. Because the static memoryless state feedback controller is concerned here, the controller can be moved to the actuator side, so we denote $\tau_{k}=\tau_{S C}^{k}+\tau_{C A}^{k}+\tau_{C}^{k}=\tau$ as a constant, and the system is under the following assumptions:

(1) The time delay is shorter than the sampling period $\tau<h_{\min }$;

(2) Time varying sampling period is $T_{k}=h_{k}+\tau$, due to $h_{k}$ is time-varying and bounded, the sampling period of the NCS is time-varying and bounded, that is $T_{k} \in\left[T_{\min }, T_{\max }\right]$;

(3) Denote $h_{0}$ as the nominal value of $h_{k}$, then $h_{k}=h_{0}+\Delta_{k}$, where $\Delta_{k}$ is the uncertain part of the timevarying sampling period;

(4) The sensors are clock-driven, the sampling instant is $t_{k}$, and the sampling period is $T_{k}=t_{k+1}-t_{k}$, the controllers and the actuators are event-driven.
The zero-order-holder $(\mathrm{ZOH})$ at the actuator side makes the $u\left(t-\tau_{k}\right)$ be the same one when $t \in\left[t_{k}, t_{k}+\tau\right)$ and $t \in\left[t_{k}+\tau, t_{k+1}\right)$, that is

$u(t)=\left\{\begin{array}{l}u\left(t_{k-1}\right), \quad t_{k} \leq t<t_{k}+\tau \\ u\left(t_{k}\right), \quad t_{k}+\tau \leq t<t_{k+1}\end{array}\right.$

Discretizing the system according to the sampling period $h_{k}$, the discrete system is obtained as follows:

$$
\begin{aligned}
& x(k+1)=\mathrm{e}^{A\left(h_{k}+\tau\right)} x(k)+\int_{k T_{k}}^{k T_{k}+\tau} \mathrm{e}^{A_{C}\left(k T_{k}+T-s\right)} \mathrm{d} s B_{C} u(k-1) \\
& +\int_{k T_{k}+\tau}^{k T_{k}+T_{k}} \mathrm{e}^{A_{C}\left(k T_{k}+T-s\right)} \mathrm{d} s B_{C} u(k) \\
& =\mathrm{e}^{A\left(h_{k}+\tau\right)} x(k)+\mathrm{e}^{A_{C} h_{k}} \int_{0}^{\tau} \mathrm{e}^{A_{C} s} \mathrm{~d} s B_{C} u(k-1) \\
& +\int_{0}^{h_{k}} \mathrm{e}^{A_{C} s} \mathrm{~d} s B_{C} u(k)
\end{aligned}
$$

\section{Denote}

$$
H=\left[\begin{array}{cc}
A_{c} & B_{c} \\
0 & 0
\end{array}\right] \text {, }
$$

where $A_{c}$ and $B_{c}$ are constant matrices of appropriate dimensions, then

$$
F(T) \triangleq \exp (H T)
$$

$=\left[\begin{array}{cc}\exp \left(A_{c} T\right) & \int_{0}^{T} \exp \left(A_{c} s\right) B_{c} \mathrm{~d} s \\ 0 & I\end{array}\right]$

Introducing the augmented vector

$$
z(k)=\left[\begin{array}{ll}
x^{\mathrm{T}}(k) & u^{\mathrm{T}}(k-1)
\end{array}\right]^{\mathrm{T}},
$$

then (3) can be formed as

$$
\begin{aligned}
& z(k+1)=\Phi\left(h_{k}\right) z(k)+\Gamma\left(h_{k}\right) u(k) \\
& \Phi\left(h_{k}\right)=\left[\begin{array}{cc}
\mathrm{e}^{A_{c}\left(h_{k}+\tau\right)} & \mathrm{e}^{A_{c} h_{k}} \int_{0}^{\tau} \mathrm{e}^{A_{c} s} \mathrm{~d} s B_{c} \\
0 & 0
\end{array}\right] \\
& =A_{0}+\left[\begin{array}{l}
I \\
0
\end{array}\right]\left[\begin{array}{ll}
\mathrm{e}^{A_{c} \Delta k}-I & \int_{0}^{\Delta k} \mathrm{e}^{A_{c} s} B_{c} \mathrm{~d} s
\end{array}\right] A_{0} \\
& =A_{0}+\varphi^{-1}\left[\begin{array}{l}
I \\
0
\end{array}\right] \varphi \int_{0}^{\Delta k} \mathrm{e}^{A_{c} s} B_{c} \mathrm{~d} s\left[\begin{array}{ll}
A_{c} & B_{c}
\end{array}\right] A_{0}=A_{0}+D F_{k} E_{1} \\
& \Gamma\left(h_{k}\right)=\int_{0}^{h_{k}} \mathrm{e}^{A_{C} s} \mathrm{~d} s B_{C} u(k) \\
& =B_{0}+\left[\begin{array}{l}
I \\
0
\end{array}\right]\left[\begin{array}{ll}
\mathrm{e}^{A_{c} \Delta k}-I & \int_{0}^{\Delta k} \mathrm{e}^{A_{c} s} B_{c} \mathrm{~d} s
\end{array}\right] B_{0}
\end{aligned}
$$


$=B_{0}+D F_{k} E_{2}$

where

$A_{0}=\left[\begin{array}{cc}\mathrm{e}^{A_{c}\left(h_{0}+\tau\right)} & \mathrm{e}^{A_{c} h_{0}} \int_{0}^{\tau} \mathrm{e}^{A_{c} s} \mathrm{~d} s B_{c} \\ 0 & 0\end{array}\right]=F\left(h_{0}\right)\left[\begin{array}{ll}I & 0 \\ 0 & 0\end{array}\right] F(\tau)$, $B_{0}=\left[\begin{array}{c}\int_{0}^{h_{0}} \mathrm{e}^{A_{c} s} \mathrm{~d} s B_{c} \\ \mathrm{I}\end{array}\right]=F\left(h_{0}\right)\left[\begin{array}{l}I \\ 0\end{array}\right]$,

$D=\varphi^{-1}\left[\begin{array}{l}I \\ 0\end{array}\right]$

$F_{k}=\varphi \int_{0}^{\Delta k} \mathrm{e}^{A_{c} s} \mathrm{~d} s$,

$E_{1}=\left[\begin{array}{ll}A_{c} & B_{c}\end{array}\right] A_{0}$,

$E_{2}=\left[\begin{array}{ll}A_{c} & B_{c}\end{array}\right] B_{0}$,

$\varphi$ is some real constant.

Then we get the discrete system of system (1) as

$$
\left\{\begin{aligned}
z(k+1) & =\left(A_{0}+D F_{k} E_{1}\right) z(k)+\left(B_{0}+D F_{k} E_{2}\right) u(k) \\
& \triangleq \bar{A} z(k)+\bar{B} u(k) \\
y(k)= & C z(k)
\end{aligned}\right.
$$

where

$\bar{A}=A_{0}+D F_{k} E_{1}$,

$\bar{B}=B_{0}+D F_{k} E_{2}$,

$C=\left[\begin{array}{ll}C_{c} & 0\end{array}\right]$.

To determine $\varphi$, the following lemmas are given.

Lemma 1. For constant matrix $A_{c},\left\|\mathrm{e}^{A_{c} t}\right\| \leq \mathrm{e}^{\eta t}$ exists for all the $t \geq 0$.

Theorem 1. The uncertain item $F_{k}$ is norm bounded, $|\Delta k| \in\left[0, \Delta_{\text {max }}\right]$, when the real constant $\varphi \neq 0$ and

$|\varphi| \leq \begin{cases}\eta /\left(\mathrm{e}^{\eta \Delta_{\max }}-1\right), & \eta \neq 0 \\ 1 / \Delta_{\max }, & \eta=0\end{cases}$

is satisfied, $F_{k}^{\mathrm{T}} F_{k} \leq I$ exists, where

$\eta=\frac{1}{2} \sigma_{\max }\left(A_{c}+A_{c}^{*}\right)$,

$\sigma_{\max }(\bullet)$ is the largest singular value, $A_{c}^{*}$ is the conjugate transpose matrix of $A_{c}$.
Proof. When $\eta \neq 0$, from lemma 1, we can get

$\| F_{k}|| \leq|\varphi| \int_{0}^{\Delta_{\max }}|| \mathrm{e}^{A t}|\mathrm{~d} t \leq| \varphi\left|\int_{0}^{\Delta_{\max }}\right|\left|\mathrm{e}^{\eta t}\right| \mathrm{d} t$

$=|\varphi|\left(\mathrm{e}^{\eta \Delta_{\max }}-1\right) / \eta$

Obviously, when $|\varphi| \leq \eta /\left(\mathrm{e}^{\eta \Delta_{\max }}-1\right)$, we obtain $F_{k}^{\mathrm{T}} F_{k} \leq I$ . When $\eta \neq 0$, the proof is omitted.

Associated with this system is the cost function

$J=\sum_{k=0}^{\infty}\left[z^{\mathrm{T}}(k) Q z(k)+u^{\mathrm{T}}(k) R u(k)\right]$

where $Q$ and $R$ are given positive-definite matrices.

For the control input $u_{i}(k), i=1,2, \cdots, m$, let $u_{i}^{F}(k)$ denote the signal from the actuator that has failed. The following failure model is adopted in this paper:

$u_{i}^{F}(k)=\alpha_{i} u_{i}(k), \quad i=1,2, \cdots, m$

where

$0 \leq \widehat{\alpha}_{i} \leq \alpha_{i} \leq \breve{\alpha}_{i}, \quad i=1,2, \cdots, m$

with $\widehat{\alpha}_{i} \leq 1$ and $\breve{\alpha}_{i} \geq 1$.

In the above model of actuator failure, if $\widehat{\alpha}_{i}=\breve{\alpha}_{i}$, then it corresponds to the normal case $u_{i}^{F}(k)=u_{i}(k)$. When $\breve{\alpha}_{i}=0$, it covers the outage case. If $\hat{\alpha}_{i}>0$, it corresponds to the partial failure case, namely, partial degradation of the actuator.

Denote

$u^{F}(k)=\left[\begin{array}{llll}u_{1}^{F}(k) & u_{2}^{F}(k) & \cdots & u_{m}^{F}(k)\end{array}\right]^{\mathrm{T}}$,

$\breve{\alpha}=\operatorname{diag}\left\{\breve{\alpha}_{1}, \breve{\alpha}_{2}, \cdots, \breve{\alpha}_{m}\right\}$,

$\widehat{\alpha}=\operatorname{diag}\left\{\widehat{\alpha}_{1}, \widehat{\alpha}_{2}, \cdots, \widehat{\alpha}_{m}\right\}$,

$\alpha=\operatorname{diag}\left\{\alpha_{1}, \alpha_{2}, \cdots, \alpha_{m}\right\}$,

$\alpha$ is said to be admissible if $\alpha$ satisfies $\widehat{\alpha} \leq \alpha \leq \breve{\alpha}$.

Define

$\beta=\operatorname{diag}\left\{\beta_{1}, \beta_{2}, \cdots, \beta_{m}\right\}$,

$\beta_{0}=\operatorname{diag}\left\{\beta_{10}, \beta_{20}, \cdots, \beta_{m 0}\right\}$

where

$\beta_{i}=\frac{\widehat{\alpha}_{i}+\breve{\alpha}_{i}}{2}, \quad \beta_{i 0}=\frac{\breve{\alpha}_{i}-\widehat{\alpha}_{i}}{\bar{\alpha}_{i}+\widehat{\alpha}_{i}}, \quad i=1,2, \cdots, m$

From (11) and (12), we define 


$$
\alpha=\left(I+\alpha_{0}\right) \beta
$$

and

$\left|\alpha_{0}\right| \leq \beta_{0} \leq I$,

where

$\alpha_{0}=\operatorname{diag}\left\{\alpha_{01}, \alpha_{02}, \cdots, \alpha_{0 m}\right\},\left|\alpha_{0}\right|=\operatorname{diag}\left\{\left|\alpha_{01}\right|,\left|\alpha_{02}\right|, \cdots,\left|\alpha_{0 m}\right|\right\}$.

Definition 1. A control law $u(k)=K z(k)$ is said to be a reliable guaranteed cost control law associated with a cost matrix $P>0$ for system (1) and cost function (8), if the following matrix inequality

$[\bar{A}+\bar{B} \alpha K]^{\mathrm{T}} P[\bar{A}+\bar{B} \alpha K]-P+Q+K^{\mathrm{T}} \alpha R \alpha K<0$

holds for all admissible $\alpha$ and uncertain matrix $F_{k}$ satisfying $F_{k}^{\mathrm{T}} F_{k} \leq I$.

Lemma 2.(Schur complement). Given the constant matrices $\Omega_{1}, \Omega_{2}, \Omega_{3}$ of appropriate dimensions, where $\Omega_{1}=\Omega_{1}^{\mathrm{T}}$ and $\Omega_{2}=\Omega_{2}^{\mathrm{T}}>0$, then

$\Omega_{1}+\Omega_{3}^{\mathrm{T}} \Omega_{2}^{-1} \Omega_{3}<0$

if and only if

$\left[\begin{array}{cc}\Omega_{1} & \Omega_{3}^{\mathrm{T}} \\ \Omega_{3} & -\Omega_{2}\end{array}\right]<0$ or $\left[\begin{array}{cc}-\Omega_{2} & \Omega_{3} \\ \Omega_{3}^{\mathrm{T}} & \Omega_{1}\end{array}\right]<0$.

Lemma 3 [34]. Given matrices $Y, H, E$ of appropriate dimensions and with $Y$ symmetric, then for all $F$ satisfying $F^{\mathrm{T}} F \leq I$ and

$Y+H F E+E^{\mathrm{T}} F^{\mathrm{T}} H^{\mathrm{T}}<0$,

if and only if there exists $\varepsilon>0$ such that

$Y+\varepsilon H H^{\mathrm{T}}+\varepsilon^{-1} E^{\mathrm{T}} E<0$.

\section{MAIN RESULTS}

The following theorem gives the reliable guaranteed cost controller for systems (1) and (8).

Theorem 2. $u(k)=K z(k)$ is a reliable guaranteed cost controller of the system (1) and the cost function (8), if there exist matrices $X>0, R_{0}>0, Y$, and scalar $\gamma>0$, such that for any admissible $\alpha$, the following matrix inequality holds:

$\left[\begin{array}{cccc}\gamma D D^{\mathrm{T}}-X & B_{0} R_{0} & A_{0} X+B_{0} \beta Y & B_{0} \\ * & -R^{-1}+R_{0} & \beta Y & 0 \\ * & * & -X & 0 \\ * & * & * & -R_{0}^{-1} \\ * & * & * & * \\ * & * & * & * \\ * & * & * & *\end{array}\right.$

$$
\left.\begin{array}{ccc}
0 & 0 & 0 \\
\left(E_{2} R_{0}\right)^{\mathrm{T}} & 0 & 0 \\
\left(E_{1} X+E_{2} \beta Y\right)^{\mathrm{T}} & X & Y^{\mathrm{T}} \beta \beta_{0} \\
E_{2}^{\mathrm{T}} & 0 & 0 \\
-\gamma I & 0 & 0 \\
* & -Q^{-1} & 0 \\
* & * & -R_{0}
\end{array}\right]<0
$$

where $(*)$ denotes the symmetric element of a matrix, and the feedback gain matrix $K$ is given by

$K=Y X^{-1}$,

moreover, the corresponding closed-loop cost function satisfies

$J \leq z_{0}^{\mathrm{T}} P z_{0}$

Proof. Define Lyapunov function

$V(z)=z^{\mathrm{T}} P z$

from Definition 1, for all the non-zero $z(k)$ and $F_{k}^{\mathrm{T}} F_{k} \leq I$, the derivative of $V(z)$ along any trajectory of the closedloop system

$$
z(k+1)=(\bar{A}+\bar{B} \alpha K) z(k)
$$

satisfies

$\Delta V(z)=V(z(k+1))-V(z(k))$

$\leq-z(k)^{\mathrm{T}}\left(Q+K^{\mathrm{T}} \alpha R \alpha K\right) z(k)<0$.

So the closed-loop system is quadratic stable. Furthermore, from (16), we can obtain

$z(k)^{\mathrm{T}} Q z(k)+u(k)^{\mathrm{T}} \alpha R \alpha u(k)<-\Delta V(z)$,

By summing both sides of the above inequality from $k=0$ to $k=\infty$, due to $z(\infty)=0$, we get

$\sum_{k=0}^{\infty}\left(z(k)^{\mathrm{T}} Q z(k)+u(k)^{\mathrm{T}} \alpha R \alpha u(k)\right) \leq z_{0}^{\mathrm{T}} P z_{0}$.

It is sufficient to show that (16) holds for all the admissible $\alpha$ and $K$ given by (18), by the Schur complement, for $P>0$, inequality (16) is equivalent to

$\left[\begin{array}{cc}-P^{-1} & \bar{A}+\bar{B} \alpha K \\ * & Q-P+K^{\mathrm{T}} \alpha R \alpha K\end{array}\right]<0$

and using Schur complement again, for $R>0$,

$\left[\begin{array}{ccc}-P^{-1} & 0 & \bar{A}+\bar{B} \alpha K \\ * & -R^{-1} & \alpha K \\ * & * & Q-P\end{array}\right]<0$ 
By (14), (15) and (20), and using the inequality

$$
2 a^{\mathrm{T}} b \leq a^{\mathrm{T}} a+b^{\mathrm{T}} b
$$

for any diagonal matrix $R_{0}>0$, it follows that

$$
\begin{aligned}
& {\left[\begin{array}{ccc}
-P^{-1} & 0 & \bar{A}+\bar{B} \alpha K \\
* & -R^{-1} & \alpha K \\
* & * & Q-P
\end{array}\right]=\left[\begin{array}{ccc}
-P^{-1} & 0 & \bar{A}+\bar{B} \beta K \\
* & -R^{-1} & \beta K \\
* & * & Q-P
\end{array}\right]} \\
& +\left[\begin{array}{c}
\bar{B} \\
I \\
0
\end{array}\right] \alpha_{0}\left[\begin{array}{lll}
0 & 0 & \beta K
\end{array}\right]+\left(\left[\begin{array}{c}
\bar{B} \\
I \\
0
\end{array}\right] \alpha_{0}\left[\begin{array}{lll}
0 & 0 & \beta K
\end{array}\right]\right)^{\mathrm{T}} \\
& \leq\left[\begin{array}{ccc}
-P^{-1} & 0 & \bar{A}+\bar{B} \beta K \\
* & -R^{-1} & \beta K \\
* & * & Q-P
\end{array}\right] \\
& +\left[\begin{array}{c}
\bar{B} \\
I \\
0
\end{array}\right] R_{0}\left[\begin{array}{c}
\bar{B} \\
I \\
0
\end{array}\right]^{\mathrm{T}}+\left[\begin{array}{c}
0 \\
0 \\
K^{\mathrm{T}} \beta
\end{array}\right] R_{0}^{-1} \beta_{0}^{2}\left[\begin{array}{c}
0 \\
0 \\
K^{\mathrm{T}} \beta
\end{array}\right]^{\mathrm{T}} \\
& =\left[\begin{array}{ccc}
-P^{-1}+\bar{B} R_{0} \bar{B}^{\mathrm{T}} & \bar{B} R_{0} & \bar{A}+\bar{B} \beta K \\
* & -R^{-1}+R_{0} & \beta K \\
* & * & Q-P+\Xi
\end{array}\right]
\end{aligned}
$$

where

$\Xi=K^{\mathrm{T}} \beta R_{0}^{-1} \beta_{0}^{2} \beta K$. ty

Thus, the inequality (20) is true if the following inequali-

$$
\left[\begin{array}{ccc}
-P^{-1}+\bar{B} R_{0} \bar{B}^{\mathrm{T}} & \bar{B} R_{0} & \bar{A}+\bar{B} \beta K \\
* & -R^{-1}+R_{0} & \beta K \\
* & * & Q-P+\Xi
\end{array}\right]<0
$$

holds. Pre- and post-multiplying the left-hand side matrix in (21) by the matrix

$\operatorname{diag}\left\{I, I, P^{-1}\right\}$,

respectively, it follows that the matrix inequality (21) is equivalent to

$$
\left[\begin{array}{ccc}
-P^{-1}+\bar{B} R_{0}{ }^{-\mathrm{T}} & \bar{B} R_{0} & \bar{A} P^{-1}+\bar{B} \beta K P^{-1} \\
* & -R^{-1}+R_{0} & \beta K P^{-1} \\
* & * & P^{-1} Q P^{-1}-P^{-1}+P^{-1} \Xi P^{-1}
\end{array}\right]<0
$$

Define $X=P^{-1}, Y=K P^{-1}$, using the Schur complement, we can get
$\left[\begin{array}{cccc}-X & \bar{B} R_{0} & \bar{A} X+\bar{B} \beta Y & \bar{B} \\ * & -R^{-1}+R_{0} & \beta Y & 0 \\ * & * & X^{\mathrm{T}} Q X-X+X^{\mathrm{T}} \Xi X & 0 \\ * & * & * & -R_{0}^{-1}\end{array}\right]<0$

Substitute

$\bar{A}=A_{0}+D F_{k} E_{1}$,

$\bar{B}=B_{0}+D F_{k} E_{2}$

into the above inequality,

$\left[\begin{array}{cccc}-X & B_{0} R_{0} & A_{0} X+B_{0} \beta Y & B_{0} \\ * & -R^{-1}+R_{0} & \beta Y & 0 \\ * & * & X^{\mathrm{T}} Q X-X+X^{\mathrm{T}} \Xi X & 0 \\ * & * & * & -R_{0}^{-1}\end{array}\right]$
$+\left[\begin{array}{c}D \\ 0 \\ 0 \\ 0\end{array}\right] F_{k}\left[\begin{array}{llll}0 & E_{2} R_{0} & E_{1} X+E_{2} \beta Y & E_{2}\end{array}\right]$
$+\left(\left[\begin{array}{l}D \\ 0 \\ 0 \\ 0\end{array}\right] F_{k}\left[\begin{array}{llll}0 & E_{2} R_{0} & E_{1} X+E_{2} \beta Y & E_{2}\end{array}\right]\right)^{\mathrm{T}}<0$

for $F_{k}^{\mathrm{T}} F_{k} \leq I$, there exists a scalar $\gamma>0$, such that

$$
\left[\begin{array}{cccc}
-X & B_{0} R_{0} & A_{0} X+B_{0} \beta Y & B_{0} \\
* & -R^{-1}+R_{0} & \beta Y & 0 \\
* & * & X^{\mathrm{T}} Q X-X+X^{\mathrm{T}} \Xi X & 0 \\
* & * & * & -R_{0}^{-1}
\end{array}\right]
$$

$+\gamma\left[\begin{array}{c}D \\ 0 \\ 0 \\ 0\end{array}\right]\left[\begin{array}{l}D \\ 0 \\ 0 \\ 0\end{array}\right]^{\mathrm{T}}$

$+\gamma^{-1}\left[\begin{array}{c}0 \\ \left(E_{2} R_{0}\right)^{\mathrm{T}} \\ \left(E_{1} X+E_{2} \beta Y\right)^{\mathrm{T}} \\ E_{2}^{T}\end{array}\right]\left[\begin{array}{c}0 \\ \left(E_{2} R_{0}\right)^{\mathrm{T}} \\ \left(E_{1} X+E_{2} \beta Y\right)^{\mathrm{T}} \\ E_{2}^{T}\end{array}\right]^{\mathrm{T}}<0$

Using the Schur complement again, we can get 


$$
\begin{aligned}
& {\left[\begin{array}{ccc}
\gamma D D^{\mathrm{T}}-X & B_{0} R_{0} & A_{0} X+B_{0} \beta Y \\
* & -R^{-1}+R_{0} & \beta Y \\
* & * & X^{\mathrm{T}} Q X-X+X^{\mathrm{T}} \Xi X \\
* & * & * \\
* & * & *
\end{array}\right.} \\
& \left.\begin{array}{cc}
B_{0} & 0 \\
0 & \left(E_{2} R_{0}\right)^{\mathrm{T}} \\
0 & \left(E_{1} X+E_{2} \beta Y\right)^{\mathrm{T}} \\
-R_{0}^{-1} & E_{2}^{\mathrm{T}} \\
* & -\gamma I
\end{array}\right]<0
\end{aligned}
$$

Using the Schur complement again, (17) is obtained. The proof is complete.

Based on Theorem 2, the problem in design of the optimal reliable guaranteed cost controller can be structured by resolving a certain optimization problem as following.

Theorem 3. Consider the system (1) with cost function (8), if the following optimization problem

$$
\begin{aligned}
& \min _{\gamma, \zeta, R_{0}, X, Y} \zeta \\
& \text { s.t. (1) }(17), \\
& \text { (2) }\left[\begin{array}{cc}
-\zeta & z_{0}^{\mathrm{T}} \\
z_{0} & -X
\end{array}\right]<0 .
\end{aligned}
$$

has a solution $\gamma, \zeta, R_{0}, X, Y$, then

$u(k)=Y X^{-1} z(k)$

is the optimal reliable guaranteed cost controller.

Proof. It is proven by the Schur complement that the constraint condition (2) in the (23) is equivalent to $z_{0}^{\mathrm{T}} X^{-1} z_{0}<\zeta$. We get the result of the theorem from Theorem 2.

\section{SIMULATION}

For the NCS

$$
\left\{\begin{array}{l}
\dot{x}(t)=\left[\begin{array}{cc}
0 & 1 \\
0 & -0.1
\end{array}\right] x(t)+\left[\begin{array}{c}
0 \\
0.1
\end{array}\right] u(t) \\
y(t)=\left[\begin{array}{ll}
0 & 1
\end{array}\right] x(t)
\end{array}\right.
$$

We take the time-varying part of the sampling period as $h_{k} \in\left[\begin{array}{ll}0.5 & 1.0\end{array}\right] \mathrm{s}$, time delay $\tau=0.4 \mathrm{~s}, Q=0.5 I_{3 \times 3}, R=1$.

Because $\eta=0.5525 \neq 0,|\varphi| \leq \eta /\left(e^{\eta \Delta_{\max }}-1\right)$. It is assumed that the input to the system has partial failure as follows: $\hat{\alpha}=0.75, \bar{\alpha}=1.25$. For the $h_{0}=0.5\left(h_{\max }+h_{\min }\right)$, we choose $\varphi=3.1$, the corresponding optimal reliable guaranteed cost controller is $u(k)=\left[\begin{array}{lll}-0.8527 & -6.6814 & -0.3958\end{array}\right] z(k)$,

the associated upper bound over the closed-loop cost function is $J^{*}=91.5973$.

\section{CONCLUSION}

For linear time-invariant controlled plant, under the assumption that the time delay is constant and shorter than the sampling period, the sampling is time-varying within a certain known bound, the system is transformed into a timevarying discrete time system, where the time-varying sampling period parts are treated as norm bounded uncertainties of the structure parameter. We have derived a sufficient condition for the existence of reliable guaranteed cost controllers by applying the linear matrix inequality (LMI) approach. Moreover, we have formulated a convex optimization problem with LMI constraints for designing the optimal reliable guaranteed cost controller which decreases the upper bound of the closed-loop system cost. We have proposed the reduction algorithms for decreasing the required amount of LMIs needed for stability analysis.

\section{CONFLICT OF INTEREST}

The authors confirm that this article content has no conflict of interest.

\section{ACKNOWLEDGEMENTS}

This work was supported by the Shandong Provincial Natural Science Foundation, China (ZR2011FQ015).

\section{REFERENCES}

[1] D. Yue, Q.-L. Han, and C. Peng, "State feedback controller design of networked control systems," IEEE Transactions on Circuits and Systems-II: Express Briefs, vol. 51, no. 11, pp. 640-644, 2004.

[2] W. Zhang, M. S. Branicky, and S. M. Phillips, "Stability of networked control systems," IEEE Control System Magazine, vol. 21, pp. 84-99, 2001.

[3] J. Feng, and S.-Q. Wang. "Reliable fuzzy control for a class of nonlinear networked control systems with time delay," Acta Automatica Sinica, vol. 38, no. 7, pp. 1091-1099, 2012.

[4] S. Wen, Z. Zeng, and T. Huang, "Robust probabilistic sampling $\mathrm{H} \infty$ output tracking control for a class of nonlinear networked systems with multiplicative noises," Journal of the Franklin Institute, vol. 350, pp. 1093-1111, 2013.

[5] Y. Shi, and B. Yu, "Robust mixed H2/Hœ control of networked control systems with random time delays in both forward and backward communication links," Automatica, vol. 47, no. 4, pp. 754-760, 2011.

[6] F. Yang, and Q.-L. Han, "Hळ control for networked sys-tems with multiple packet dropouts," Information Sciences, vol. 252, pp. 106$117,2013$.

[7] W. Chen, and L. Qiu, "Stabilization of networked control systems with multirate sampling," Automatica, vol. 49, no. 6, pp. 1528-1537, 2013.

[8] Y. Wang, and G. Yang, "Hळ controller design for networked control systems via active-varying sampling period method," Acta Automation Sinica, vol. 34, no. 7, pp. 814-818, 2008.

[9] R. A. Borges, R. C. Oliveira, and C. T. Abdallah, "Robust Hळ networked control for systems with uncertain sampling rates," Control Theory \& Applications, vol. 4, no. 1, pp. 50-60, 2010. 
[10] H. Gao, J. Wu, and P. Shi, "Robust sampled-data $\mathrm{H} \infty$ control with stochastic sampling," Automatica, vol. 45, no. 7, pp. 1729-1736, 2009.

[11] L. Hetel, J. Daafouz, and C. Iung, "Analysis and control of LTI and switched systems in digital loops via an event-based modeling," International Journal of Control, vol. 81, no. 7, pp. 1125-1138, 2008.

[12] F. Liu, Y. Yao, and F. He, "Stability analysis of networked control systems with time-varying sampling periods," Journal of Control Theory and Application, vol. 6, no. 1, pp. 22-25, 2008.

[13] M. Izák, D. Görges, and S. Liu, "Stabilization of systems with variable and uncertain sampling period and time delay," Nonlinear Analysis: Hybrid Systems, vol. 4, pp. 291-305, 2010.

[14] Y. Xu, H. Su, and Y.-J. Pan, "Output feedback stabilization for markov-based nonuniformly sampled-data networked control systems," Systems \& Control Letters, vol. 62, pp. 656-663, 2013.

[15] G.-Y. Tang, "Suboptimal control for nonlinear systems: a successive approximation approach," Systems and Control Letters, vol. 54, pp. 429-434, 2005.

[16] G.-Y. Tang, and H.-H. Wang, "Successive approximation approach of optimal control for nonlinear discrete-time systems," International Journal of Systems Science, vol. 36, pp. 153-161, 2005.

[17] D. Yue, and Q.-L. Han, "Delayed feedback control of uncertain systems with time-varying input delay," Automatica, vol. 41, pp. 233-240, 2005.

[18] D. Yue, "Robust stability of uncertain systems with unknown input delay," Automatica, vol. 40, pp. 331-336, 2004.

[19] Q.-L. Han, "Robust stability of uncertain delay-differential systems of neutral type," Automatica, vol. 38, pp. 719-723, 2002.

[20] Q.-L. Han, "On robust stability of neutral systems with timevarying discrete delay and norm-bounded uncertainty," Automatica, vol. 40, pp. 1087-1092, 2004.

[21] S. Chang, and T. Peng, "Adaptive guaranteed cost control of systems with uncertain parameters," IEEE Translation and Automation Control, vol. 17, pp. 474-483, 1972.
[22] I. Petersen, and D. McFarlane, "Optimal guaranteed cost control and filtering for uncertain linear systems," IEEE Transaction and Automation Control, vol. 39, pp. 1971-1977, 1994.

[23] N. Xie, and G.-Y. Tang, "Delay-dependent nonfragile guaranteed cost control for nonlinear time-delay systems," Nonlinear Analysis, vol. 64, pp. 2084-2097, 2006.

[24] L. Yu, and F. Gao, "Optimal guaranteed cost control of discretetime uncertain systems with both state and input delays," Journal of Franklin Instittute, vol. 338, pp. 101-110, 2001.

[25] L. Yu, "An LMI approach to reliable guaranteed cost control of discrete-time systems with actuator failure," Applied Mathematics and Computation, vol. 162, pp. 1325-1331, 2005.

[26] J. Wang, and H. Shao, "Delay-dependent robust and reliable $\mathrm{H} \infty$ control for uncertain time-delay systems with actuator failures," Journal of the Franklin Institute, vol. 337, pp. 781-791, 2000.

[27] S. Takahide, "Network system, controller, and network control method," U.S. Patent 8611220, 2013.

[28] N. Akio, "Communication network control system and control method," U.S. Patent 8,422,359, 2013.

[29] V.S.M. Richardus, V.H. Robert, and V.M. Henricus, "Networked control system using logical addresses," U.S. Patent 8,218,547, 2013.

[30] I. Taro, "Network connection control system," U.S. Patent $8,335,156,2012$.

[31] S. Masayuki, F., Takahiro, and T. Erika, "Network management station, network control system, and network management method," U.S. Patent 8,477,601, 2013.

[32] N. Akira, O. Tetsuya, and O. Ryutaro, "Network control system," U.S. Patent 8,396,060, 2013.

[33] T. Osamu, A. Shuichi, I. Yoshiyuki, and M. Takayuki, "Network control device and network control method," U.S. Patent 8,447,334, 2013.

[34] B.R. Barmish, "Necessary and sufficient conditions for quadratic stability of an uncertain system," Journal of Optimization Theory and Applications, vol. 46, pp. 399-408, 1985.

(C) Xie and Xia; Licensee Bentham Open.

This is an open access article licensed under the terms of the Creative Commons Attribution Non-Commercial License (http://creativecommons.org/licenses/by-nc/3.0/) which permits unrestricted, non-commercial use, distribution and reproduction in any medium, provided the work is properly cited. 